\title{
Aleitamento materno: uso da tecnologia da informação como estratégia para a construção de um website
}

Breastfeeding: Use of Information Technology as a strategy for the creation of a website* Lactancia materna: uso de la Tecnología de la Información como estrategia para diseñar un sitio web

\section{Myrcea Nunes Dellalibera', Débora Fernandes Coelho ${ }^{\text {II }}$}

\begin{abstract}
Resumo: Objetivo: descrever o processo de criação e desenvolvimento de um website focado em orientações de aleitamento materno. Método: estudo envolvendo a construção de um produto do Mestrado Profissional de Enfermagem, com finalidade de comunicação em saúde. Para a elaboração do site foi utilizado o método Definition, Architecture, Design and Implementation. Resultados: construi-se um website com conteúdos sobre aleitamento materno, com linguagem simples, inclusiva e de fácil entendimento. A utilização de figuras e vídeos permitiu um design atrativo e uma organização intuitiva. Conclusão: o produto foi elaborado e embasado a partir das melhores evidências científicas, de forma sitematizada e organizado de maneira lógica, a fim de promover uma navegação fácil e didática.
\end{abstract}

Descritores: Aleitamento materno; Tecnologia da informação; Internet; Redes de comunicação de computadores; Educação em saúde

\begin{abstract}
Objective: to describe the process to create and develop a website focused on breastfeeding guidelines. Method: a study involving the creation of a product of the Professional Master's Degree in Nursing, focused on communication in health. The Definition, Architecture, Design and Implementation method was used to create the website. Results: a website was created with content on breastfeeding, using simple, inclusive and easy-to-understand language. The use of figures and videos allowed for an appealing design and an intuitive organization. Conclusion: the product was elaborated and based on the best scientific evidence, in a systematized and logical manner, in order to promote easy and didactic navigation.
\end{abstract}

Descriptors: Breastfeeding; Information technology; Internet; Computer communication networks; Health education

Resumen: Objetivo: describir el proceso para crear y desarrollar un sitio web enfocado en pautas de lactancia materna. Método: estudio que implicó la creación de un producto de la Maestría Profesional de Enfermería, con el propósito de

\footnotetext{
${ }^{\text {I }}$ Enfermeira, Mestre em Enfermagem-UFCSPA, Especialista Materno-Infantil-FCSMV, Universidade Federal de Ciências da Saúde de Porto Alegre. Porto Alegre, RS, Brasil. E-mail: myrcea.dellalibera@gmail.com, Orcid: http://orcid.org/0000-0001-9417-3823

II Enfermeira obstétrica, Doutora em Enfermagem-UFRGS, Mestre em Enfermagem-UFRGS, Especialista em Enfermagem em cuidado prénatal-UNIFESP, Especialista em gênero e sexualidade - UERJ, Universidade Federal de Ciências da Saúde de Porto Alegre. Porto Alegre, RS, Brasil. E-mail: deborafe@ufcspa.edu.br, Orcid: https://orcid.org/0000-0002-4535-2611

* Extraído da dissertação “Aleitamento Materno: A construção de um website para apoio, promoção e proteção”, Programa de Pós-Graduação em Enfermagem, Universidade Federal de Ciências da Saúde de Porto Alegre, 2020.
} 
Aleitamento materno: uso da tecnologia da informação como estratégia para a construção... | 2

facilitar la comunicación en materia de salud. Para elaborar el sitio web se empleó el método Definition, Architecture, Design and Implementation. Resultados: se creó un sitio web con diversos contenidos sobre lactancia materna, con idioma sencillo, inclusivo y fácil de entender. El uso de figuras y videos permitió lograr un diseño atractivo y una organización intuitiva. Conclusión: el producto se elaboró sobre la base de las mejores evidencias científicas disponibles, en forma sistematizada y con organización lógica, a fin de promover una navegación sencilla y didáctica.

Descriptores: Lactancia materna; Tecnología de la información; Internet; Redes de comunicación de computadores; Educación en salud

\section{Introdução}

Diante do panorama mundial, o aleitamento materno é um tema que vem sendo muito pesquisado no decorrer dos últimos anos. Isso se deve ao fato de estudos comprovarem seus benefícios à mulher e a criança. A amamentação poderia prevenir a morte de 823.000 crianças menores de cinco anos e 20.000 mortes de mulheres por câncer de mama anualmente em todo o mundo, independente da classe social. ${ }^{1}$

A Organização Mundial da Saúde (OMS) e Ministério da Saúde (MS) incentivam a prática do aleitamento. A recomendação é que todas as crianças iniciem a amamentação na primeira hora de vida e sejam amamentadas exclusivamente até os seis meses de idade. Após esse período, o aleitamento deve ser mantido e complementado com alimentos saudáveis até dois anos ou mais. ${ }^{2}$

No entanto, os índices de aleitamento materno ainda estão abaixo do que é recomendado pela OMS. No último estudo realizado para analisar a tendência dos indicadores da prática dos últimos 34 anos, os resultados preliminares mostraram um avanço nas taxas de exclusividade em crianças menores de seis meses, na qual o percentual foi de $45,7 \%$. Isso demonstra um aumento de 4,7 pontos percentuais em 22 anos quando comparado a última pesquisa brasileira em $2008 .^{3}$

Buscando melhores desfechos e, assim, objetivando a redução das taxas de desnutrição e mortalidade infantil, muitas estratégias têm sido usadas como ações de apoio e incentivo à prática. As políticas públicas de saúde foram criadas para reforçar, adequar, ampliar e integrar intervenções e estratégias relacionadas ao aleitamento materno. Estas ações são realizadas por profissionais de saúde qualificados envolvidos no cuidado materno-infantil e, apesar de todos os esforços para a 
promoção de sua exclusividade até os seis meses de vida da criança, sabe-se que as dificuldades encontradas no processo são muitas. Crenças, a falta de preparo da mulher no retorno ao trabalho, influência de práticas inadequadas, a industrialização e criação de produtos considerados impróprios, e a influência do marketing sobre alimentos artificiais são fatores que contribuem com essas dificuldades. ${ }^{4-5}$

Além disso, sabe-se que a influência das mídias sociais atualmente são de elevada importância no que diz respeito a informação. A Internet proporciona a divulgação da informação de forma rápida, compreendendo um amplo número de pessoas, possibilitando uma construção de conhecimento coletivo. Facilita a participação no processo de aprendizado e propicia a mudança de comportamentos. Ressaltam-se as tecnologias da informação, como ferramentas capazes de gerar interesse das pessoas sobre o tema. ${ }^{6-7}$

Estudos incluídos em uma revisão integrativa indicam que o apoio profissional juntamente com a educação em saúde e o uso de tecnologias da informação trazem contribuições positivas no aumento das taxas de aleitamento materno e o enfermeiro tem um papel fundamental nessa criação de novas tecnologias. ${ }^{6}$ As ferramentas desenvolvidas a partir das tecnologias da informação para a saúde vêm como importante complemento do cuidado assistencial ao paciente. Os profissionais de saúde, em especial os enfermeiros, estão cada vez mais desenvolvendo e aplicando esses recursos na área materno-infantil com o objetivo de promoção e educação à mulher e sua rede de apoio.

Assim como a internet pode influenciar positivamente com informações baseadas em evidências, também pode ocorrer a divulgação de informações inadequadas e sem qualidade. Por meio de visita a alguns sites disponíveis on-line e abordando a mesma temática, observou-se que há uma carência de informações de qualidade e excesso de páginas contendo informações baseadas na experiência própria, como blogs e troca de experiências com outras mães.

A amamentação é um período de insegurança e dúvidas. O intuito dessa construção acadêmica é fornecer para as mulheres que amamentam e sua rede de apoio um meio de adquirir 
Aleitamento materno: uso da tecnologia da informação como estratégia para a construção... | 4

conhecimento de forma rápida, fácil e confiável. O estudo objetivou descrever o processo de criação e desenvolvimento de um website focado em orientações de aleitamento materno.

\section{Método}

Trata-se de um estudo metodológico de produção tecnológica que envolveu o desenvolvimento de um produto do Mestrado Profissional de Enfermagem, direcionado a construção de um website com conteúdos e orientações sobre aleitamento materno baseado em evidências científicas. O estudo foi realizado na Universidade Federal de Ciências da Saúde de Porto Alegre nos anos de 2019 e 2020. Para a etapa de construção do website contou-se com a colaboração de um acadêmico do curso de Informática Biomédica da referida Universidade.

Para a elaboração do website, foi necessário o uso de um método que permitisse o acesso as informações de maneira organizada e estruturada que atendesse diferentes leitores. Este método é um conjunto de normas, procedimentos, técnicas e ferramentas de análise que definem o padrão desejado para o planejamento de sistemas ou aplicações educacionais. ${ }^{8}$ Foi escolhido para a construção do produto o método DADI (Definition, Architecture, Design and Implementation), que possibilita uma melhor organização quanto a divisão de trabalho, a reunião de informações, a escolha do layout e a implementação da programação. É composto por quatro etapas: definição, arquitetura, design e implementação. ${ }^{7}$

$\mathrm{Na}$ etapa "definição" foram estipulados os objetivos, o público-alvo e os conteúdos, isto é, foi realizado um planejamento de todos os assuntos e recursos necessários para o desenvolvimento do website. O objetivo do site definido foi fornecer informações, orientações e atualizações em aleitamento materno. O público-alvo escolhido foram gestantes, mulheres que amamentam, sua rede de apoio e pessoas que tenham interesse no tema, como profissionais da saúde e estudantes.

Quanto aos conteúdos, foi realizado uma busca on-line para identificar o referencial teórico com relevância sobre o tema proposto. Para o embasamento teórico do conteúdo do website 
utilizou-se manuais de atenção básica do MS, Guia Alimentar para crianças brasileiras menores de 2 anos, assim como livro-texto e artigos sobre aleitamento materno., ${ }^{2,9-10} \mathrm{Na}$ segunda etapa, definida como "arquitetura" foi realizado a estruturação dos conteúdos e o planejamento da interatividade com o usuário e a navegabilidade, a fim de facilitar o acesso ao conteúdo.

A organização dos conteúdos foram realizados de acordo com a sua importância e hierarquia. $\mathrm{Na}$ arquitetura da informação, os temas foram agrupados, identificados e separados em quatro categorias principais. Estas, foram dispostas nas opções de menu na seguinte forma: "conhecendo a mama”, "amamentando", "problemas na amamentação" e "acessórios para a amamentação”. Além destas páginas, há também a página “início”, “sobre este site” e "atualidades”.

A etapa “design” é a criação do website, na qual as informações foram organizadas e estruturadas de forma que facilitasse a visualização do website. Nesta fase ocorreu a escolha do tipo e tamanho das fontes, imagens, vídeos, paleta de cores e a revisão dos textos. Optou-se por utilizar a ferramenta "Google Sites", devido a facilidade de acesso e opção de gratuidade, sem ônus a pesquisadora e aos usuários. Outro fator de escolha desta ferramenta foi a atratividade do site que possibilita uma experiência de ensino e aprendizagem de maneira mais dinâmica e atraente. Os conteúdos foram dispostos em cada página, mantendo uma relação com as figuras, fotos e vídeos para uma melhor compreensão do assunto, evitando uma possível confusão de interpretação. Os textos foram escritos com uma linguagem simples, de fácil entendimento e buscando, o máximo possível, a utilização de uma linguagem inclusiva.

A última etapa do método, foi a "implementação", esta etapa diz respeito a testagem de todas as funções, verificação dos links, vídeos, navegabilidade e interface. Foi realizada a apresentação do website para opinião pública de profissionais especialistas na área materno-infantil com ênfase em aleitamento materno por meio de uma videoconferência. Estavam presentes no encontro quatro enfermeiras atuantes na área materno-infantil, uma nutricionista, a orientadora do trabalho - enfermeira obstetra e um acadêmico do Curso de Informática Biomédica. O encontro 
Aleitamento materno: uso da tecnologia da informação como estratégia para a construção... | 6

teve duração de duas horas e meia e consistiu na opinião pública das especialistas sobre o layout e conteúdos do website. Na discussão do grupo foram sugeridas revisões dos textos explicativos para uma perspectiva de linguagem inclusiva, como também a diversificação das imagens, representando as mais variadas raças que representavam as pessoas. Outra sugestão foi a inserção de vídeos explicativos sobre os temas, como opção para pessoas com deficiência visual. Após o encontro com especialistas, procedeu-se a finalização e teste de todas as páginas e, seguindo, após, para o lançamento com divulgação do website na rede. Nesta etapa, também se realizou o monitoramento do website por meio da ferramenta Google Analytics.

Este trabalho não foi submetido ao Comitê de Ética em Pesquisa, por não se tratar de pesquisa direta com seres humanos, conforme art. 1-- item VII da Resolução 510/16. Respeitou as exigências éticas e científicas, baseadas na Resolução 510/16, e na Lei 9.610/98 da Casa Civil..11-12

\section{Resultados}

O website resultou em um documento de sete páginas, com 47 figuras, 10 fotos e oito vídeos. O site nomeado "Aleitamento Materno" pode ser acessado por meio do seguinte link: https://sites.google.com/view/aleitamentomaterno. ${ }^{13}$

A página inicial do website contém informações referentes ao contexto do aleitamento materno, suas recomendações e benefícios, e atualidades do tema. Nesta página, o leitor têm a possibilidade de comunicar-se com a autora por meio de um formulário. Clicando no item "Enviar mensagem” abrirá uma página com um formulário para escrever comentários, dúvidas e sugestões.

A segunda página do website é a página "Sobre este site". Nela, contém informações como: objetivos, público-alvo, justificativa, autoria e colaboração. Um vídeo de apresentação do site no início da página foi produzido pela autora, com o objetivo de explicar o que se encontrará no site.

A terceira, quarta, quinta e sexta páginas referem-se aos conteúdos específicos sobre 
aleitamento materno. Em todas as páginas frases importantes foram destacadas em cartazes ilustrativos para chamar a atenção do leitor. O website ainda traz na última aba, denominada atualidades, alguns assuntos relacionados ao tema, como banco de leite humano e atualizações e orientações específicas sobre COVID-19 e amamentação. Ainda, há um espaço chamado "Palavra de Especialista”, no qual um vídeo realizado por uma especialista e consultora internacional de aleitamento materno dá dicas sobre o tema.

O monitoramento do site, foi realizado por meio da funcionalidade Google Analytics, que permite a visualização e o acompanhamento das estatísticas de visitação ao website, como também variáveis demográficas, tipo de recurso utilizado para acessar, entre outros. No período de um mês do lançamento do site na internet, obteve-se 620 sessões. As sessões são o número total de visitas que obteve-se dentro do período estipulado para o monitoramento. O número de usuários que entraram no website uma vez dentro do período analisado foi de 430. A duração média das sessões (visitas) foi de 02 minutos e 54 segundos. Este valor mostra o tempo que o visitante gastou no site. Já o número de visualizações de páginas foi de 1.794 , isto representa a média do número de páginas que um visitante acessou no site.

Em relação à faixa etária dos usuários que acessaram o site, pode-se destacar que dos 191 usuários identificados, 69 (36\%) estavam entre 25 e 34 anos de idade, seguido de 35 a 44 anos com 34 pessoas (17,8\%). Em relação ao sexo, foi possível identificar 197 usuários, na qual 132 (67\%) eram femininos e 65 (33\%) pessoas do sexo masculino. Verificou-se que dos 430 usuários identificados, $393(91,4 \%)$ dos acessos foram realizados no Brasil, porém tiveram acessos também em outros países, $24(5,6 \%)$ nos Estados Unidos, $5(1,16 \%)$ na Itália, $3(0,70 \%)$ na Espanha, $1(0,23 \%)$ no Canadá, 1 na Austrália, 1 na Colômbia, 1 na França e 1 no Reino Unido.

Outro item analisado foi o tipo de dispositivo usado para acessar o site pelo usuário, constata-se que dos 430 usuários identificados na plataforma Google Analytics, 340 (79\%) utilizou o celular, $85(19,77 \%)$ usou o computador e $5(1,16 \%)$ utilizaram o tablet como dispositivo para acesso. 
Aleitamento materno: uso da tecnologia da informação como estratégia para a construção... | 8

Salienta-se que foi desenvolvido um site intuitivo, de fácil navegabilidade também responsivo, que possibilita o acesso por diversos aparelhos.

\section{Discussão}

Atualmente, a internet possibilita, por meio da criação de websites a transmissão de conhecimento baseado em evidências científicas e experiência profissional do pesquisador, possibilitando uma acessibilidade rápida das pessoas. Cada vez mais o uso de tecnologias da informação vem sendo utilizada como estratégia de prática educativa para apoio, promoção e proteção ao aleitamento materno pelos enfermeiros que atuam nessa área. Por isso, por meio dos recursos disponíveis no meio tecnológico, o enfermeiro tem a possibilidade de promover de forma eficiente e abrangente o conhecimento construído para o público-alvo, por meio do ensino, da pesquisa ou cuidado assistencial. ${ }^{14}$

Existem uma infinidade de websites desenvolvidos de forma não estruturada, com informações confusas e difícil acessibilidade. Buscou-se trazer no website informações sobre todo o processo que envolve a amamentação, incluindo cuidados baseados em manuais do MS e Sociedade Brasileira de Pediatria. Os conteúdos foram selecionados e após categorizados por temas para facilitar a leitura e compreensão das informações pelos usuários. Procurou-se usar uma linguagem simples, inclusiva e informal para a abrangência de todos os públicos. A informação disponibilizada de forma simples e de fácil entendimento, melhora a expectativa e a possibilidade de aquisição de conhecimento. Isso possibilita que o público-alvo desenvolva sua capacidade de autonomia e habilidade, estimulando-os a ter uma mudança de comportamento na medida em que ele se torna sujeito do seu próprio desenvolvimento intelectual. ${ }^{15}$

$\mathrm{Na}$ aba inicial é possível visualizar todos os temas disponíveis por meio da barra superior e também nos ícones inseridos na parte inferior. Conforme indicado, o rigor metodológico deve ser priorizado quando se pensa em desenvolver um website, pois os conteúdos disponibilizados para a 
população devem ser fornecidos de forma adequada e acessível. ${ }^{16}$

O website aborda temas como anatomia da mama, hormônios da amamentação, descida do leite, tipos de mamilos, pega correta, posicionamento para amamentar, problemas durante a amamentação, acessórios mais utilizados pelas mulheres, banco de leite e atualidades como o novo coronavírus. A escolha desses temas vem ao encontro do que tem escrito na literatura como principais necessidades de informação na temática como, também, a partir da experiência profissional das autoras.

Em relação a duração média das visitas ao site, o tempo foi de 02 minutos e 54 segundos. Este valor mostra o tempo que o visitante gastou no site. Pesquisa aponta que atualmente as pessoas ficam on-line cerca de 6 horas e 43 minutos diariamente. ${ }^{17}$ Tendo em vista o resultado deste estudo, foi considerado pequeno o tempo gasto no site.

Quanto aos acessos ao site observou-se que as mulheres (67\%) foram as que mais acessaram. No entanto, houve acesso por homens (33\%) também, demonstrando uma mudança na atitude por parte destes. A participação do parceiro no processo de aleitamento materno ainda precisa ser estimulado e incentivado. O suporte fornecido à mulher no período lactacional pelo parceiro tem fundamental importância para o sucesso da prática. Isso se deve ao fato que o apoio emocional paterno é visto como um fator de proteção para a mulher no período gravídico-puerperal, auxiliando-a a enfrentar os desafios relacionados as alterações emocionais, fisiológicas e sociais. ${ }^{18}$ No entanto, pode-se perceber que os homens estão tendo um maior interesse pelo tema abordado, seja para oferecer um auxílio mais qualificado a sua parceira ou assumir cuidados com o bebê.

Em relação a idade dos leitores, ficou evidente que a maioria das pessoas que acessou o site estão na faixa etária de maior fertilidade. O que demonstra o maior interesse desse público em informar-se sobre questões da amamentação.

O tipo de dispositivo mais usado pelas pessoas para acessar o website foi celular. Pesquisa aponta que no Brasil o uso do celular está em ascensão, sendo o principal meio de acesso a internet 
Aleitamento materno: uso da tecnologia da informação como estratégia para a construção... | 10 no país. Em 2019, o celular foi o dispositivo mais usado por pessoas que tinham acesso a internet. Entre os anos de 2017 e 2018, houve um aumento das pessoas que acessaram a internet por meio do celular (passou de 97\% para 98,1\%). Devido a esse crescente uso do celular nos últimos anos, outros equipamentos estão deixando de serem usados. O uso de computadores caiu de 56,6\% para 50,7\% e de tablets de $14,3 \%$ para $12 \%$ no mesmo período. ${ }^{19}$

A divulgação se deu por meio de páginas das redes sociais da Universidade, Conselho de Enfermagem (COREN-RS) e plataformas de mensagens instantâneas privadas. Segundo o Instituto Brasileiro de Geografia e Estatística (IBGE), é alto o número de pessoas que tem acesso a internet e utilizam a rede para comunicação por mensagens de texto, mensagens de voz, visualização de vídeos e imagens. ${ }^{20}$

É evidente a crescente busca de conhecimentos por meio digital como websites, mídias sociais e aplicativos. Estas plataformas possibilitam um acesso imediato e atualizado de informações de qualidade relacionadas a educação em saúde. Desta forma, as tecnologias da informação incluem a mulher, sua rede de apoio e os profissionais de saúde na construção do processo de ensino-aprendizagem..$^{21-22}$

O produto de mestrado profissional em enfermagem foi concluído de acordo com o objetivo e método propostos. As limitações da pesquisa foram o tempo hábil para o monitoramento do website, o baixo número de acessos e a validação do site por usuários e também especialistas da área. Neste âmbito, sugere-se a realização de novos estudos que avaliem e validem o uso de websites na educação em saúde.

Como contribuições para a área da Enfermagem e Educação em saúde, destacou-se a construção de um website, que possibilitou o acesso a informações de qualidade sobre aleitamento materno para pessoas que amamentam e interessados no tema. 


\section{Conclusão}

As tecnologias em saúde são um importante instrumento de aprendizado que se constituem em uma fonte de divulgação rápida e ampla das informações. Existe a necessidade de oferecer as pessoas que amamentam e interessadas no tema, uma fonte de informação com orientações atualizadas e de qualidade. Portanto, o website foi construido e embasado a partir das melhores evidências científicas, de forma sistematizada e organizada de maneira lógica, a fim de facilitar a navegação e localização dos conteúdos.

Cada vez mais as pessoas têm acesso rápido à internet, portanto a criação de websites objetiva fornecer conhecimento e orientações de forma acessível, fácil e confiável para um melhor entendimento do processo de amamentação. Recomenda-se a continuidade deste estudo com a validação do website por especialistas da área e, além disso, avaliar o impacto no processo de fornecimento de informação e desenvolvimento de conhecimentos por parte dos usuários do site. Espera-se que, com a maior divulgação deste trabalho, possam surgir novas iniciativas de educação em saúde por meio da internet, visto que estas vêm para complementar e enriquecer as ações realizadas de forma presencial.

\section{Referências}

1. Victora CG, Barros AJD, França GVA, Bahl R, Rollins NC, Horton S, et al. Amamentação no século 21: epidemiologia, mecanismos, e efeitos ao longo da vida. Epidemiol Serv Saúde. 2016; 387(3):1-24.

2. Ministério da Saúde (BR). Saúde da Criança: aleitamento materno e alimentação complementar. Brasília (DF): Ministério da Saúde; 2015. (Cadernos de Atenção Básica).

3. Universidade Federal do Rio de Janeiro (UFRJ). Estudo Nacional de Alimentação e Nutrição Infantil ENANI-2019: resultados preliminares: indicadores de aleitamento materno no Brasil [Internet]. Rio de Janeiro: UFRJ; 2020 [acesso em 2020 ago 20]. Disponível em: https://enani.nutricao.ufrj.br/index.php/relatorios/

4. Ministério da Saúde (BR), Secretaria de Atenção à Saúde, Departamento de Ações Programáticas Estratégicas. Bases para a discussão da Política Nacional de Promoção, Proteção e Apoio ao Aleitamento 
Aleitamento materno: uso da tecnologia da informação como estratégia para a construção... | 12

Materno. Brasília (DF): Ministério da Saúde, 2017.

5. Silva DSS, Oliveira M, Souza ALTD, Silva RM. Promoção do aleitamento materno: políticas públicas e atuação do enfermeiro. Cad UniFOA. 2017;35:135-40.

6. Silva AC, Freitas LMC, Maia JAF, Granja MMF, Dodt RCM, Chaves EMC. Tecnologias em aleitamento materno: revisão integrativa. Rev Bras Promoç Saúde. 2016;29(3):439-46.

7. Silva NVN, Pontes CM, Sousa NFC, Vasconcelos MGL. Tecnologias em saúde e suas contribuições para a promoção do aleitamento materno: revisão integrativa da literatura. Ciênc Saúde Colet. 2019;24(2):589-602. doi: 10.1590/1413-81232018242.03022017

8. Falkembach GAM. Concepção e desenvolvimento de material educativo digital. Renote. 2005; 3(1):1-15.

9. Ministério da Saúde (BR), Secretaria de Atenção Primaria à Saúde, Departamento de Promoção da Saúde. Guia alimentar para crianças brasileiras menores de 2 anos. Brasília (DF): Ministério da Saúde; 2019.

10. Carvalho MR, Gomes CF. Amamentação: bases científicas. 4ํe ed. Barueri (SP): Guanabara Koogan; 2017.

11. BRASIL. Conselho Nacional de Saúde. Resolução nº 510, de 07 de abril de 2016. Dispõe sobre as normas aplicáveis a pesquisas em Ciências Humanas e Sociais cujos procedimentos metodológicos envolvam a utilização de dados diretamente obtidos com os participantes ou de informações identificáveis ou que possam acarretar riscos maiores do que os existentes na vida cotidiana. Diário Oficial da União: seção 1, Brasília, DF, n. 98, p. 44-46, 24 maio 2016.

12. BRASIL. Lei no 9.610, de 19 de fevereiro de 1998. Altera, atualiza e consolida a legislação sobre direitos autorais e dá outras providências. Diário Oficial da União: seção 1, Brasília, DF, n. 36, p. 11-17, 20 fev. 2008.

13. Dellalibera MN, Coelho DF. Aleitamento Materno [Internet]. Produto do Mestrado Profissional em Enfermagem da Universidade Federal de Ciências de Saúde de Porto Alegre (UFCSPA). Porto Alegre: UFCSPA; 2020. Disponível em: https://sites.google.com/view/aleitamentomaterno/in\%C3\%ADcio

14. Lins TH, Marin HF. Avaliação de website sobre assistência de enfermagem na sala de recuperação pósanestésica. Acta Paul Enferm. 2012;25(1):109-15. doi: 10.1590/S0103-21002012000100019

15. Lima FAA, Galiza FT, Silva ARV, Beserra EP, Medeiros JRR, Lima MA. Tecnologia e educação em saúde: avaliação de um website para o ensino de oftalmologia. Rev Enferm Atual In Derme. 2016; 79(17):70-5. doi: 10.31011/reaid-2016-v.79-n.17-art.340

16. Guimarães CMS, Imamura ME, Richter S, Monteiro JCS. Amamentação e tecnologias mHealth: análise dos aplicativos móveis para tablets e smartphones. Rev Eletrônica Enferm. 2018;20:v20a28.

17. Kepios. Digital 2020: Brazil [Internet]. 2020 Feb 17 [cited 2020 Apr 15]. Available from: https://datareportal.com/reports/digital-2020-brazil

18. Cavalcanti TRL, Holanda VR. Participação paterna no ciclo gravídico-puerperal e seus efeitos sobre a saúde da mulher. Enferm Foco. 2019;10(1):93-8. doi: 10.21675/2357-707X.2019.v10.n1.1446

19. Instituto Brasileiro de Geografia e Estatística (IBGE). Pesquisa Nacional por Amostra de Domicílios Continua - PNAD Contínua [Internet]. Rio de Janeiro: IBGE; 2020 [acesso em 2020 set 30]. Disponível em: 
https:/www.ibge.gov.br/estatisticas/sociais/populacao/17270-pnad-continua.html?edicao=27138\&amp;t=oque-e

20. Instituto Brasileiro de Geografia e Estatística (IBGE). Pesquisa Nacional por Amostra de Domicílios Continua - PNAD Contínua - Acesso à Internet e posse de telefone móvel celular para uso pessoal 2018 [Internet]. Rio de Janeiro: IBGE; 2020 [acesso em 2020 set 30]. Disponível em: https://biblioteca.ibge.gov.br/visualizacao/livros/liv101705_informativo.pdf

21. Dalmaso MS, Bonamigo AW. A pesquisa on-line sobre amamentação: entre o senso comum e a OMS na era digital. RECIIS. 2019;13(4):911-21. doi: 10.29397/reciis.v13i4.1635

22. Rocha ALA, Góes FGB, Pereira FMV, Moraes JRMM, Barcia LLC, Silva LF. O processo de ensinoaprendizagem de puérperas nutrizes sobre aleitamento materno. Rev Cuid. 2018;9(2): 2165-76. doi: 10.15649/cuidarte.v9i2.510

Editora Científica Chefe: Cristiane Cardoso de Paula

Editora associada: Aline Cammarano Ribeiro

\section{Autor correspondente}

Myrcea Nunes Dellalibera

E-mail: myrcea.dellalibera@gmail.com

Endereço: Rua Oito de Julho, 95 Porto Alegre-RS CEP: 90690-240

\section{Contribuições de Autoria}

1 - Myrcea Nunes Dellalibera

concepção ou desenho do estudo/pesquisa, análise e/ou interpretação dos dados, revisão final com participação crítica e intelectual no manuscrito.

\section{2 - Débora Fernandes Coelho}

Análise e/ou interpretação dos dados, revisão final com participação crítica e intelectual no manuscrito.

\section{Como citar este artigo}

Dellalibera MN, Coelho DF. Breastfeeding: use of information technology as a strategy for the creation of a website. Rev. Enferm. UFSM. 2021 [Accessed on: Year Month Day]; vol.11 e55: 1-13. DOI: https://doi.org/10.5902/2179769264034 